\title{
Two-stage Particle Swarm Optimization-based Nonlinear Model Predictive Control Method for Reheating Furnace Process
}

\author{
Zhenhao TANG ${ }^{1,2,3)}$ and Yang YANG ${ }^{2,3) *}$ \\ 1) State Key Laboratory of Synthetical Automation for Process Industries, Northeastern University, Shenyang, 110819 China. \\ 2) Liaoning Key Laboratory of Manufacturing System and Logistics, Northeastern University, Shenyang, 110819 China. \\ 3) The Logistic Institute, Northeastern University, Shenyang, 110819 China.
}

(Received on September 4, 2013; accepted on May 18, 2014)

\begin{abstract}
The steel slab temperature control of reheating furnace process plays an important role in the production of high quality reheated slab. Because of the characteristics of nonlinearity, long time-delay and uncertainty, high-accuracy slab temperature control is a challenging problem. This paper proposes a twostage particle swarm optimization (PSO)-based nonlinear model predictive control (NMPC) method to solve the problem. In this method support vector machine (SVM) is utilized to construct the nonlinear predictive model based on the real production data. To obtain better predictive model dynamically, PSO optimizes the parameters of SVM for different problems. Then PSO solves the rolling optimization problem in NMPC to obtain the proper control variables. Finally, the production data collected from a real reheating furnace process are utilized to test the proposed method. Numerical experiments are done by computer simulation based on the real production data. The experiment results illustrate that the PSO-based SVM can obtain accurate predictive model. Moreover, the proposed nonlinear model predictive control method can obtain outstanding control accuracy in steel slab temperature control.
\end{abstract}

KEY WORDS: steel slab temperature control; particle swarm optimization; nonlinear model predictive control; reheating furnace.

\section{Introduction}

Reheating furnace process heats steel slabs before hot rolling process and the heating quality influences the quantity and quality of the hot rolling products significantly. To meet the requirements of high quality products, the steel slab temperature control must be precise. However, there is no accurate mechanism model for the steel slab temperature in reheating furnace so far. Additionally, the reheating process has the properties of nonlinearity, long time-varying, uncertainty and strong coupling. ${ }^{1)}$ Thus the control of the steel slab temperature in reheating furnace is a practical and challenging problem.

For the reheat furnace process, there have been some interesting results, ${ }^{2-4)}$ however, most of them focus on the scheduling level rather than the control level. The researches of reheating process control mostly focus on furnace temperature control. Traditional PID control method is utilized widely in furnace temperature control. Many other control methods, such as decoupling control, ${ }^{5)}$ artificial neural network control, ${ }^{1,6)}$ predictive control, ${ }^{7)}$ nonlinear model predictive control ${ }^{8)}$ and a control method based on heat conduction equation $^{9)}$ are utilized in furnace temperature control. All these researches intend to control the reheating quality of steel slabs, and one of the most important quality factors is

\footnotetext{
* Corresponding author: E-mail: yyang@tli.neu.edu.cn
} DOI: http://dx.doi.org/10.2355/isijinternational.54.1836 slab temperature. But the steel slab temperature cannot be obtained with non-destructive testing methods, which lead to the former researches controlling the steel slab temperature indirectly. In this paper, we control the steel slab temperature directly through a nonlinear model predictive control method.

For the solution strategy, evolutionary algorithm is widely used in the optimization of steel production management, ${ }^{10,11)}$ however, it is limited in our problem since our problem cannot be directly formulated by deterministic model. The model predictive control (MPC) proposed in 1990s contains many methods, such as model algorithm control (MAC), ${ }^{12}$ dynamic matrix control (DMC), ${ }^{13)}$ and autoregressive moving average model based generalized predictive control (GPC). ${ }^{14)}$ These MPC methods contain three main parts: the predictive model, the rolling optimization and the feedback correction. These methods obtain good control results in many fields, such as chemical process, ${ }^{15,16)}$ sewer network control, ${ }^{17)}$ power system control, ${ }^{18)}$ and benchmark problems. ${ }^{19)}$ Even some of them are utilized commercially. But most of these methods are based on linear model which does not fit for the nonlinear problems such as the problem in this paper. To solve the nonlinear problems, nonlinear MPC is proposed. In the nonlinear MPC method, the predictive model can be utilized on the basis of the model that can predict the target accurately.

In this paper, because the impulse response model utilized in MAC cannot be obtained and the mechanism model of 
the steel slab temperature ${ }^{20)}$ cannot be utilized in MPC directly, the data-driven model based on support vector machine (SVM) proposed by Vapnik in $1995^{21)}$ is utilized to obtain predictive model. Particle swarm optimization (PSO) proposed by Kennedy \& Eberhart ${ }^{22)}$ is an iteration-based global optimization algorithm. It provides good global search ability in addressing nonlinear problem. To obtain a high-accuracy predictive model, PSO is employed to optimize the parameters of SVM. Then, to solve the rolling optimization problem with nonlinear model in NMPC, PSO is utilized once more in rolling optimization part. Moreover, the feedback correction part intends to improve the accuracy of the predictive model, and in this paper a threshold value is set to reconstruct the predictive model to keep the predictive accuracy at a high level. Additionally, the SVM predictive model utilized in this paper is optimized by PSO to improve the predictive accuracy.

In conclusion, we proposed a two-stage particle swarm optimization-based nonlinear model predictive control (TPSONMPC) method to address the steel slab temperature control problem. To solve the nonlinear problems in this method, PSO is utilized in both the predictive model optimization and the rolling optimization. The experiments based on practical production data are designed to testify the proposed method. The results demonstrate that the optimized predictive model can obtain predictive output accurately and the SVM-based model predictive control is effective on steel slab temperature control.

The rest of this paper is organized as follows. Section 2 presents the process of reheating furnace and the method for the slab temperature control. In section 3, we provide the proposed SVM-based model predictive control method. In section 4 , the proposed method is evaluated by a real production data. Finally, the conclusion is shown in section 5.

\section{Reheating Furnace Control and Particle Swarm Optimization}

In this section, we first present a description of reheating furnace process. Then we give a brief description of the approach for slab temperature control.

\subsection{Reheating Furnace Process}

Reheating furnace is an important production process in steel mill. The steel slabs which come from continuous casting or slab yard are reheated to a specified outlet temperature to meet the requirement of hot-rolling process. Additionally, the temperature rise curve and the temperature distribution must meet the technological requirements. Based on the production process, the reheating furnace is divided into four sections as shown in Fig. 1.

In the preheating section, the steel slab is heated by the exhaust steam to make use of the heat retained in the exhaust steam. The temperature of the steel slab rises rapidly in the heating section one and two. Based on the radiation heating theory, the core temperature rises behind the surface temperature. To balance the temperature distribution of steel slab, the steel slab is heated in the soaking section. Then, the heated steel slabs are transported to the hot-rolling line for further processing.

The most important quality criteria of reheating furnace

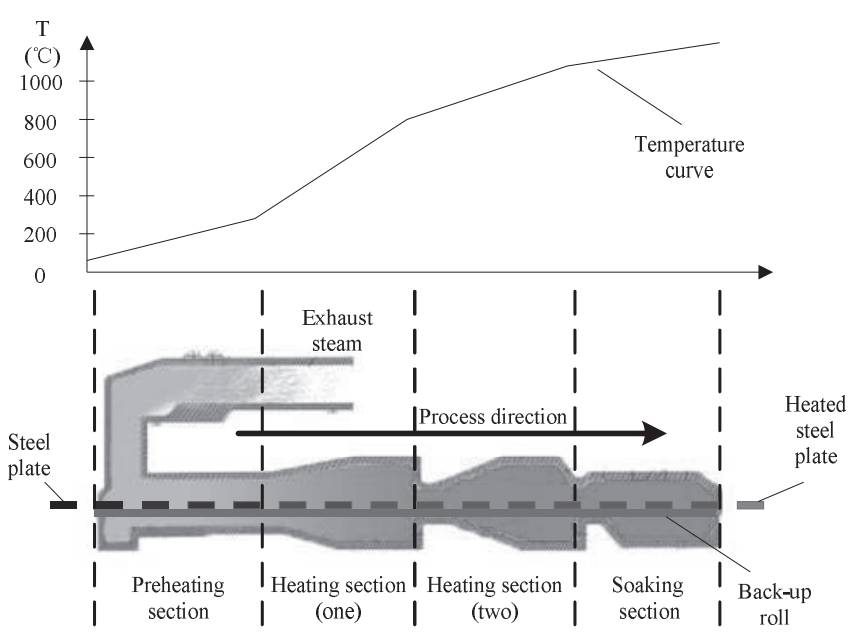

Fig. 1. Reheating furnace and temperature rise curve of steel slab.

are the outlet temperature of the steel slab surface, the temperature distribution and the oxidation ratio. All these criteria are closely related to the steel slab temperature. Thus, one of the most important purposes of reheating furnace control is the steel slab temperature control. To control the steel slab temperature, the first challenge is to inspect the steel slab temperature. Because of the heat and other environmental factors in the furnace, there is no non-destructive technology that can obtain the steel slab furnace accurately. But the buried thermocouple test data provide the foundation of obtaining the steel slab temperature by data-driven technology.

\subsection{Slab Temperature Control}

In the reheating furnace process, classical PI\PID approach or other variants are widely utilized. But the setting and adjustment of these methods are dependent on the experience of engineers. Because every reheating furnace has its features, the control methods are different from each other. Additionally, the control accuracy is unstable and these methods are inconvenient to extend to the other furnaces.

The bottleneck of utilizing advance control technology is how to obtain accurate steel slab temperature model in the reheating furnace. To solve this problem, the data-based SVM predictive model is utilized in this paper. After obtaining the steel slab temperature model, we utilize the nonlinear model predictive control to control the steel slab temperature. Model predictive control mainly consists of three parts: the predictive model, the rolling optimization and the feedback correction. Based on the difference of the predictive model, there are three predictive methods were applied widely such as model algorithm control, dynamic matrix control and generalized predictive control. The impulse response model or step response model is needed in model algorithm control. But the experiments to obtain the models will influence the regular production rhythm. Also the accurate dynamical model utilized in dynamic control cannot be obtained at present due to the strong nonlinearity of reheating production process. Different from these two methods, the model utilized in the generalized predictive control is more convenient with less constraint. Hence the nonlinear model predictive control with support vector machine model is utilized in this paper. 


\subsection{Particle Swarm Optimization}

As mentioned before, PSO is an efficient iteration optimization algorithm proposed in $1995 .{ }^{22)}$ Before the PSO is utilized, the optimization problem must be constructed. The reason is that the particles in the PSO consist of the control variables in the optimization problem, and the objective function in the optimization problem is utilized to calculate the fitness of the particles. The flowchart of PSO is shown in Fig. 2. In the initialization section, multiple particles are initialized randomly to cover the search space as wide as possible. Then, the fitness of each particle is calculated based on the particle information. If the termination condition is met, the algorithm is ended and the optimization results are outputted. Otherwise, the particles which are updated based specified formula (like (2)\&(3) in this paper) intend to obtain better control variables, then the fitness of new particles are obtained. The iteration repeats until the termination condition is met. The size of population and the factors in update formulas influence the convergence rate and the optimization accuracy significantly. These parameters are usually determined by the trial-and-error method which is utilized in this paper.

\section{TPSONMPC}

This section describes the TPSONMPC method proposed in this paper in detail. For convenience, the framework of the proposed algorithm is given in section 3.1. The major parts of the algorithm, the predictive model and the rolling optimization are given in section 3.2 and section 3.3 respectively. Then, the feedback correction is described in section 3.4.

\subsection{The Framework of TPSONMPC}

The framework of the proposed algorithm is shown in Fig. 3.

To control the steel slab temperature, the SVM predictive model is constructed. The predictive model is utilized to show the relationship between input and output of the control object. The predictive model is obtained by PSO-based SVM algorithm. Then an optimization problem based on the predictive model is constructed to minimize the control

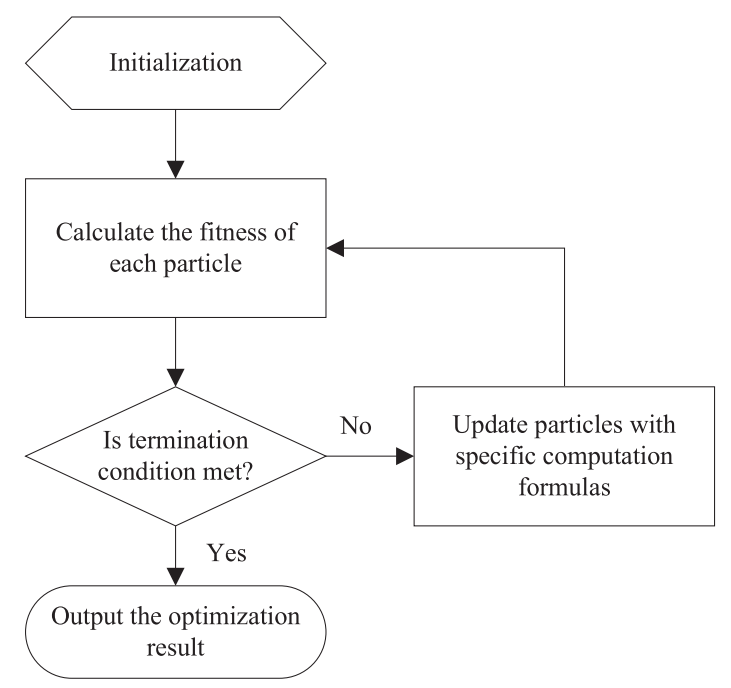

Fig. 2. The flowchart of PSO. errors between the output and the reference curve in the rolling optimization. The optimization results are the control variables $u(t)$. To guarantee the predictive accuracy, the feedback correction is utilized to modify the predictive model.

\subsection{Predictive Model}

The reheating of steel slab is a complicate process with characteristics of high nonlinearity and dynamics. Thus we utilize the SVM regression algorithm to construct the predictive model. SVM was proposed in 1995 by Vapnik. Although it was utilized in classification problems at first, it shows outstanding generalization ability in regression problems.

In the SVM algorithm, the problem of constructing a predictive model is transformed into a quadratic programming (QP) problem. The solution of the QP problem is unique and optimal. Additionally, the selection of support vectors can reduce the computation complexity and improve the generalization ability of predictive model.

Assume that the train data set is $T D=\left\{\left(\vec{x}_{i}, y_{i}\right)\right\}, i=1,2$, $\ldots$, num_s, where num_s denotes as the number of samples, $\vec{x}_{i} \in R^{\text {num }{ }_{-}{ }^{\text {in }}}$ denotes as input variables, num_in denotes as the number of input variables, $y_{i}$ denotes as the output target (in this paper means the steel slab temperature). Then the trained predictive model is $f(\vec{x})=\sum_{i \in S V} \alpha_{i} k\left(\vec{x}_{i}, \vec{x}\right)+b$, where $S V$ denotes as the dataset of support vector, $a_{i}$ and $b$ denote the coefficients obtained by solving QP problems, $\vec{x}$ denotes the new input which is needed to predict the output, and $k\left(\vec{x}_{i}, \vec{x}\right)=\phi\left(\vec{x}_{i}\right) \cdot \phi(\vec{x})$ is the kernel function.

The kernel function provides SVM an outstanding ability of addressing nonlinear problem. The selection of its parameters affects the prediction accuracy significantly. Thus the selection of the SVM parameters is treated as an optimization problem and solved by PSO in this paper.

PSO is a widely utilized swarm-based iterative optimization method. In this method, many random initial solutions are constructed as particles. After evaluating these particles by fitness, all particles fly towards the particle with best fitness. Thus, the swarm converges to the optimal solution by iterations. PSO can obtain an optimized solution rapidly with a strong global search ability which meets the requirements of model optimization problem.

The widely utilized radial basis function (RBF) kernel is utilized in this paper. In this kernel, the width parameter $\sigma$ influences the performance of the kernel significantly. Meanwhile, another parameter named penalty factor " $C$ " also influence the model accuracy significantly. The penalty factor is an intermediate variable of SVM which is utilized

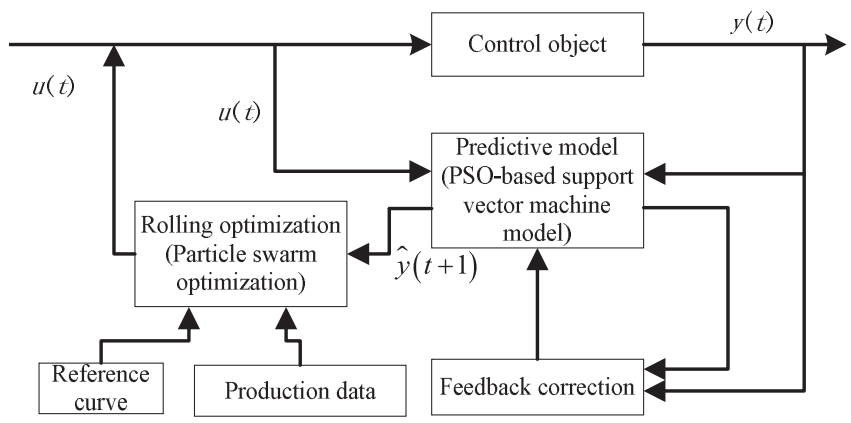

Fig. 3. The framework of TPSONMPC. 
to deal with non-separable cases. When its value is large (such as 1000), it means that few training samples are allowed to be misclassified. The predictive model is accurate in training phase, but the accuracy of the model will be unacceptable in test phase. If its value is too small, the prediction accuracy of the SVM model will be too low, and the prediction model will be meaningless. So it is an important and challenge problem to determine these two parameters. To obtain proper kernel for different problem, PSO is utilized to optimize these parameters in this paper.

The optimization problem is shown in (1).

$$
\begin{array}{ll}
\text { min. } & \sum_{h \in T \text { Test }}\left|\hat{y}_{h}-y_{h}\right| \\
\text { s.t. } & \hat{y}_{h}=f\left(\vec{x}_{h}\right) ; \\
& f\left(\vec{x}_{h}\right)=\sum_{i \in S V} \alpha_{i} k\left(\vec{x}_{i}, \vec{x}_{h}\right)+b ; \\
& k\left(\vec{x}_{i}, \vec{x}_{h}\right)=\exp \left(-\frac{\left\|\vec{x}_{i}-\vec{x}_{h}\right\|^{2}}{2 \sigma^{2}}\right) ; \\
& \sigma \geq 0 ; C \geq 0 .
\end{array}
$$

Where Test denotes as the dataset utilized to test the trained predictive model; $\hat{y}_{h}$ denotes as the predict value of $\vec{x}_{h} ; y_{h}$ denotes as the real output value of $\vec{x}_{h}$. In this problem, $\sigma$ and $C$ are the decision variables.

The flowchart of PSO-based SVM algorithm is shown in Fig. 4. Every particle consists of $\sigma$ and $C$.

To initialize PSO parameters and particles in this section, we assume that $\vec{p}_{\text {num }_{-} p, g}=(\sigma, C)$ is a particle, where num $p=$ $1,2, \ldots, N$ denotes as the number of particles, and $g=1,2$, $\ldots, G$ denotes as the generation of particles. $\vec{v}_{\text {num } p, g} \in R^{2}$ denotes as the velocity of $\vec{p}_{\text {num }} p, g$. Thus, the particles are initialized and their velocities are generated randomly within constrains. Also, the parameters $(c 1, c 2, w, G)$ utilized in PSO were initialized, $g=1$.

The termination conditions in this algorithm are 1) the relative average prediction error is smaller than $1 \%$, or 2 ) the iterations reach a specified number $G$. If one of these two conditions is met, the algorithm stops.

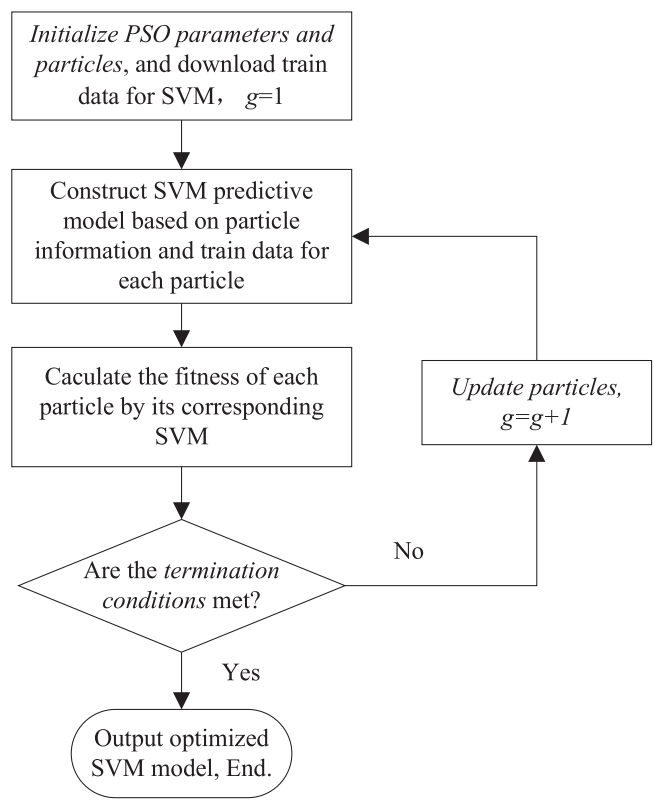

Fig. 4. The flowchart of PSO-based SVM modeling.
To update particles, the following equations are utilized.

$$
\begin{aligned}
& \vec{v}_{\text {num }_{-} p, g+1}= \\
& w \cdot\left[\vec{v}_{n u m_{-} p, g}+c_{1} \cdot r_{g} \cdot\left(\overrightarrow{p l b}_{\text {nun }_{-} p, g}-\vec{p}_{\text {num }_{-} p, g}\right)\right. \\
& \left.+c_{2} \cdot r_{g} \cdot\left(\overrightarrow{p g b}-\vec{p}_{\text {num_p }, g}\right)\right] \\
& \vec{p}_{\text {num }_{-} p, g+1}=\vec{p}_{\text {num }_{-} p, g}+\vec{v}_{\text {num }_{-} p, g+1}
\end{aligned}
$$

Where $w, c_{1}, c_{2}$ mean to control particle update velocity, $r_{g}$ is a random number between $[0,1] . \vec{v}_{n u m_{-} p, g+1}$ denotes the velocity of the num pth particle in the $g+1$ th generation. $\overrightarrow{p l b}_{n u n_{-} p, g}$ denotes the best position that the num $p$ th particle has been located till current iteration. $\overrightarrow{p g b}$ denotes the best position that all particles have been located till current iteration.

The output SVM model including support vectors, optimization parameters is saved in database for further usage.

In the model predictive control method, we need to consider the outputs of $M$ sampling times. Thus, $M$ predictive models need to be constructed for one problem.

\subsection{Rolling Optimization}

The rolling optimization in model predictive control is a local optimization. At every sampling time, a local optimization problem needs to be solved. As time passed, many local optimizations provide a good control strategy. The local optimization which is different from traditional global optimization focuses on the optimization within certain predictable time. The optimized operation variables will be utilized in production process online. Thus the optimization process must be done very quickly. Because of the high nonlinearity of predictive model, the PSO algorithm is utilized to solve the rolling optimization problem.

The objective function utilized in the rolling optimization is minimizing errors between the reference curve and the predictive output. The mathematical expression of rolling optimization is shown in (4).

$$
\begin{aligned}
& \text { min. } \sum_{j=1}^{M} \sum_{k=1}^{P}\left|\hat{y}_{j, k}-r_{j, k}\right| \\
& \text { s.t. } \hat{y}_{j, k}=f_{j, k}(\vec{u}), \\
& \quad 0 \leq \vec{u} \leq \overrightarrow{\text { Max_u}}
\end{aligned}
$$

Where $M$ denotes as the prediction length, $P$ denotes as the number of outputs. $\hat{y}_{j, k}$ denotes as the $j t h$ predictive output at $k t h$ time. $r_{j, k}$, denotes as the $j t h$ reference output at $k t h$ time. $\hat{y}_{j, k}=f_{j, k}(\vec{u})$ denotes as the model obtained in section 3.2. $\overrightarrow{M a x_{-} u}$ denotes as the upper bound of control variables. In this problem, $\vec{u}$ is the control variable vector which needs to be decided.

The steps of utilizing a PSO to solve the rolling optimization problem are given as follows.

Step 1. Initialize the particles in a PSO algorithm, assume $u_{\text {num } \_, g} \in R^{\text {num_in }}($ num $p=1,2, \ldots, N)$, num_in denotes as the number of particle dimensions which is the same as the number of control variables, $g$ denotes as the generation of the particles, the parameters $(c 1, c 2, w, G)$ utilized in the PSO were initialized, $g=1$;

Step 2. Utilize the predictive model constructed in section 3.2 and the particle information to obtain the fitness of the 
corresponding particle by (4);

Step 3. If the best fitness is smaller than 0.01 or the number of iterations reached G, go to step 5; if not, go to step 4;

Step 4. Renew the particles, check the new particles; if they satisfy the constrains, $g=g+1$, go to step 2; otherwise, regenerate the particle randomly until the constrains are satisfied, $g=g+1$, then go to step 2;

Step 5. Output the best fitness and the corresponding control variables, terminate the procedure.

\subsection{Feedback Correction}

The common feedback correction strategy is adding a weighted error in current prediction value. It works well when using the linear models. Because the predictive model is a high-nonlinearity model, we utilize a remodel strategy to improve the predictive model as the feedback correction. Hence, we set up an upper bound of prediction error to trigger a remodeling process.

Here, if five continuous prediction errors of outlet temperature are bigger than $e$ (specified before running the algorithm), the prediction model is remodeled with current data.

\section{Experiment Design and Analysis}

To testify the proposed TPSONMPC method, we design experiments with real production data. In section 4.1 and

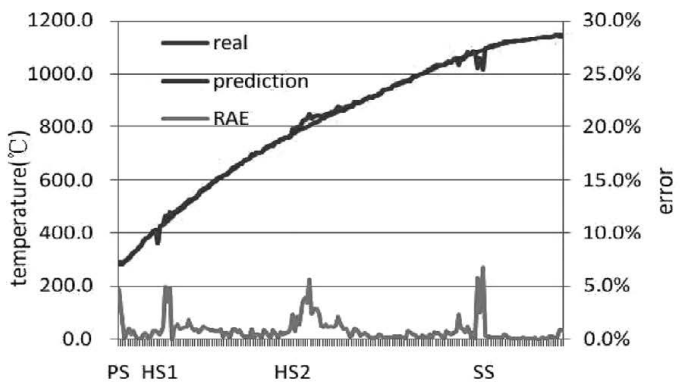

1) The prediction result of surface temperature
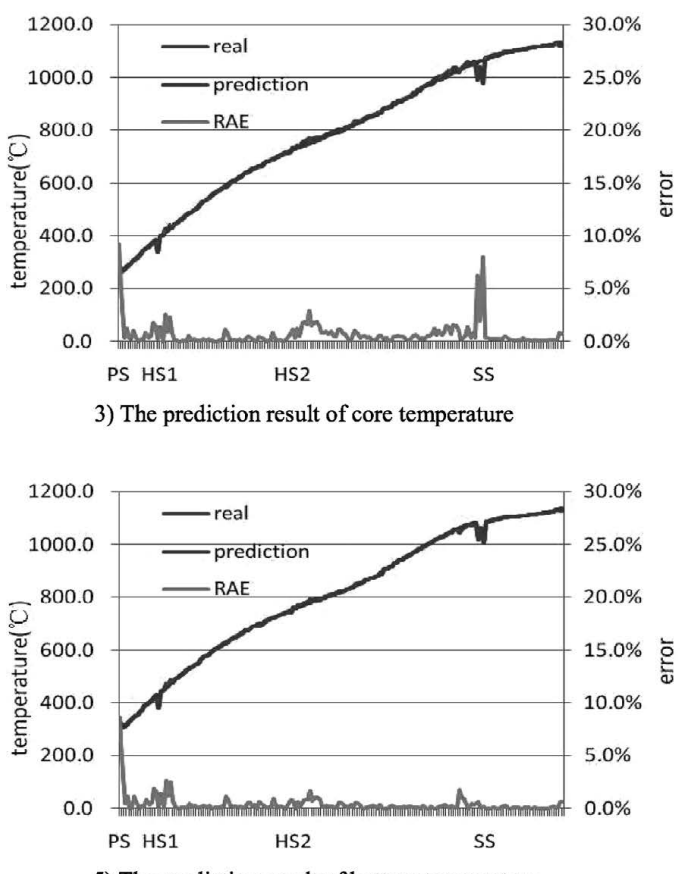

5) The prediction result of bottom temperature
4.2, the experiment environment and detailed experiment data are given respectively. The parameter setting is given in section 4.3. The experiment results and detailed analysis are shown in section 4.4 and 4.5 respectively.

\subsection{Experiment Environment}

All the experiments were done in the PC with CPU 3.00 $\mathrm{GHz}$ (dual-core) and RAM 1.99 GB. The software utilized to implement the proposed algorithm is Visual $\mathrm{C}++6.0$.

\subsection{Experiment Data}

The data utilized in the experiments were obtained from a large Chinese steel mill. The detail of the data is shown in Table 1. The number of outputs is 5 . The five outputs are the temperature of the steel slab surface, the steel slab uppermiddle, the steel slab core, the steel slab lower-middle, and

Table 1. The details of experiment data.

\begin{tabular}{cccc}
\hline data name & $\begin{array}{c}\text { Number of } \\
\text { Instances }\end{array}$ & $\begin{array}{c}\text { Number of } \\
\text { Input }\end{array}$ & $\begin{array}{c}\text { Number of } \\
\text { Output }\end{array}$ \\
\hline preheating section (PS) & 46 & 7 & 5 \\
heating section 1 (HS1) & 144 & 7 & 5 \\
heating section 2 (HS2) & 180 & 7 & 5 \\
soaking section (SS) & 90 & 7 & 5 \\
\hline
\end{tabular}

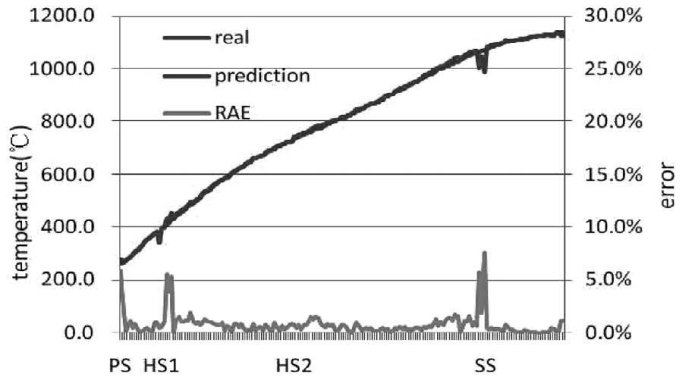

2) The prediction result of upper-middle temperature

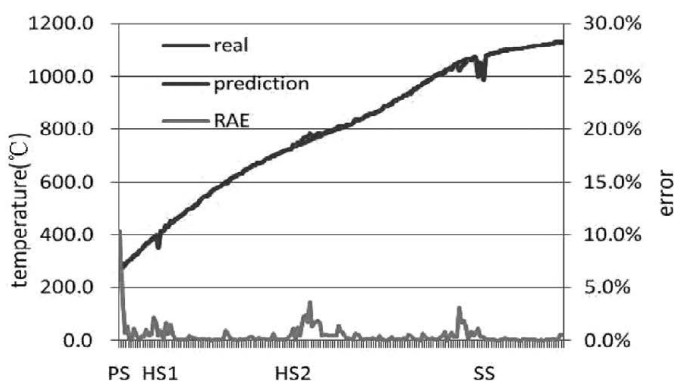

4) The prediction result of lower-middle temperature

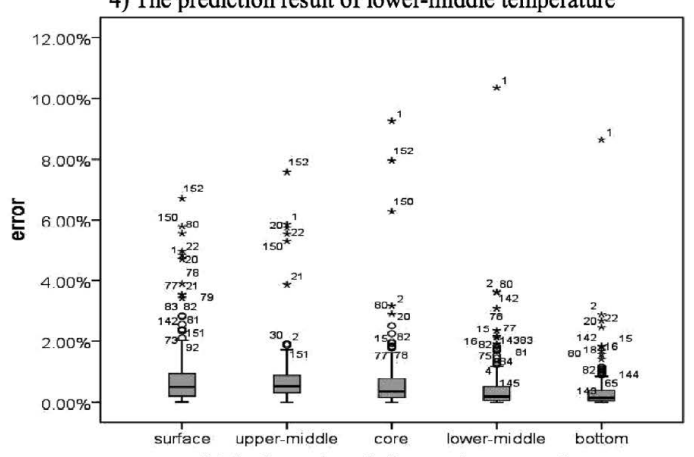

6) The box-plot of all experiment result

Fig. 6. The experiment result of the accuracy of predictive model based on SVM. 
the steel slab bottom (shown in Fig. 5).

\subsection{Parameter Selection}

The parameters need to be specified in the proposed method are PSO parameters. The RBF kernel function is utilized in this paper. The kernel parameter and penalty factor utilized in the SVM are obtain by PSO.

The PSO parameters are obtained by trail-and-error method. $N=60, G=500, w=0.7, c 1=c 2=1.414$.

Considering the requirement of production operation, the upper bound of error $e$ is set as $5 \%$.

\subsection{Experiment Results}

To testify the proposed algorithm, the experiment is done

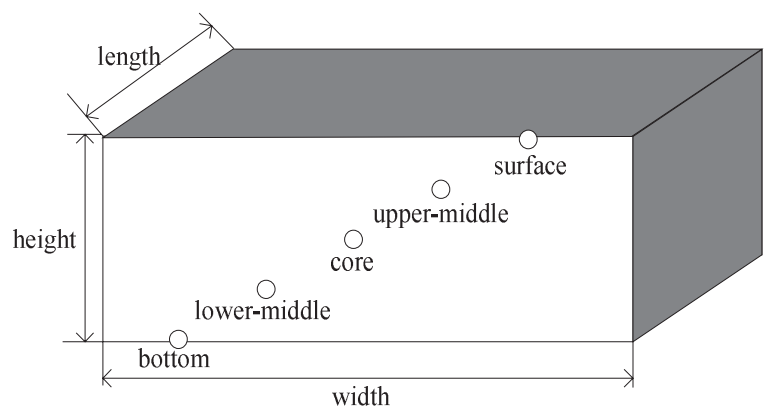

Fig. 5. The cross section of steel slab.

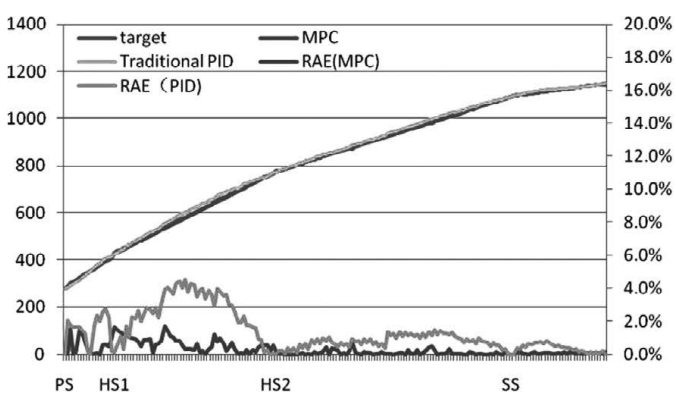

a) The control result of surface temperature

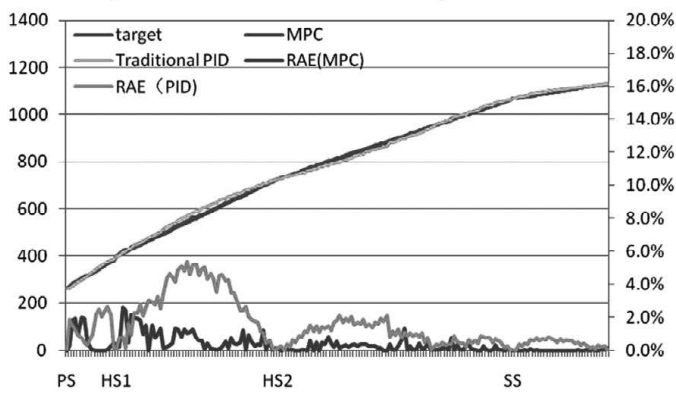

c) The control result of core temperature

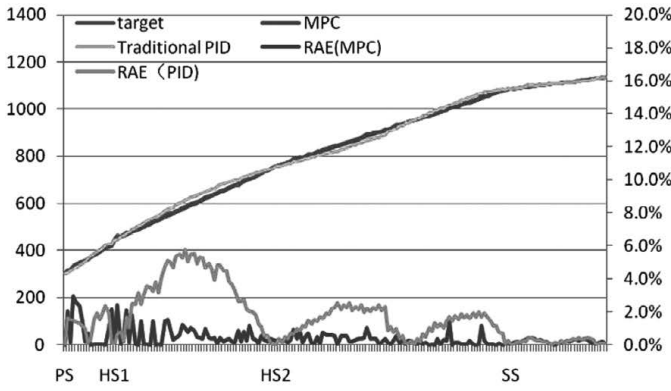

e) The control result of bottom temperature in two stages. First, the predictive ability of PSO-based SVM is testified. Then, the control accuracy is verified by the real production data.

The accuracy of SVM predictive model

To verify the effectiveness of SVM based on PSO, the accuracy of proposed model based on real production data is done. The experiment results are shown in Fig. 6.

From figures 1)-5), we can find out that: 1) the prediction results of five temperature measuring points are very close to the real value, which means that the prediction model is effective; 2) the biggest errors comes out at the points where two section are connected, such as the beginning of soaking section (SS). The major reason is that the situation in this point is very complex. That is, the input of prediction model is obtained in current section while the support vector utilized in the model is obtained in former section. So the error is much bigger than other times.

From the statistic results in figure 6), most of the errors are under 3\% except some situations. The bottom temperature predictive model performs best in five predictive models, while the surface temperature performs most unstable. The major reason is that the flame in the reheating furnace is located at the top, thus the surface temperature is affected significantly. The major heating method of other temperature measuring points is conductive heating, which is much more stable.

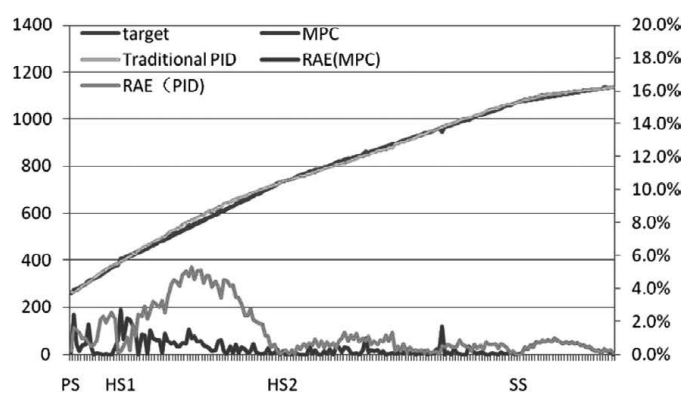

b) The control result of upper-middle temperature
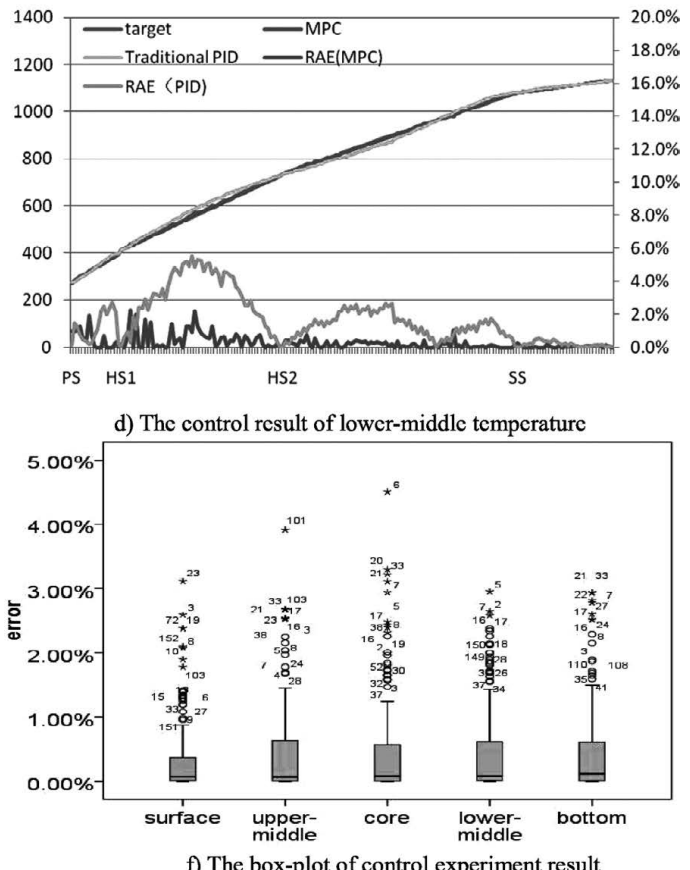

f) The box-plot of control experiment result

Fig. 7. The experiment result of the TPSONMPC and traditional PID. 


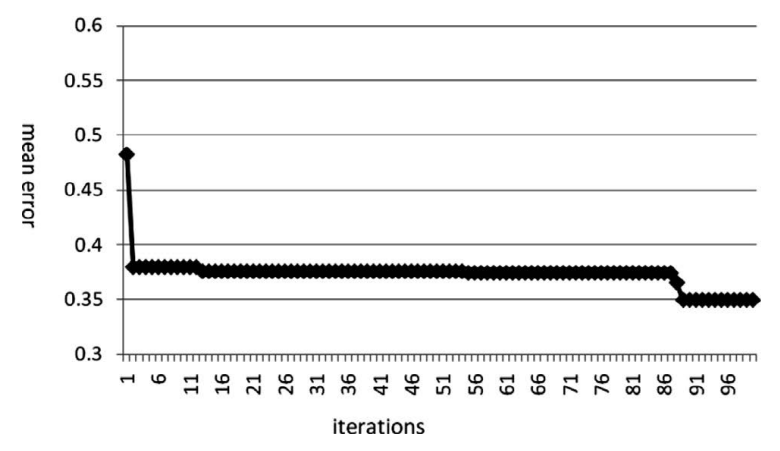

Fig. 8. The convergence curve of PSO algorithm.

\section{Control result of TPSONMPC}

The control results of the proposed MPC and the traditional PID control method are shown in Fig. 7.

From the results in figures a)-e), we can find out that 1) the control accuracy of all five temperature measure points are very high, and the MPC results follow the specified target curves accurately; 2) similar to the prediction result, the biggest error comes out at the points where two section are connected. The major reason is that the prediction error of predictive model leads to big control error. 3) Compared with the traditional PID method, the proposed MPC method produce better stability, especially in the connection part of two adjacent sections.

From the results in figure f), we can find out that most of the control error of temperature is less than $3 \%$; even the biggest error is less than 5\%. These results demonstrate that the proposed nonlinear MPC method proposed in this paper is effective.

Additionally, the convergence curve of PSO is shown in Fig. 8. The result illustrates that the control error of proposed method can reach a low level in a short time.

\subsection{Analysis of the Experiment Results}

From the result obtained in section 4.4 the following results can be summarized.

1) Predictive accuracy: The PSO-based SVM can obtain good performance in prediction accuracy. The main reason is that the PSO can determine proper parameters for the SVM algorithm dynamically in different problems.

2) Control accuracy: The control results of all five outputs meet the requirements in both control accuracy and response speed. The main reason is that the reference curve utilized in model predictive control provides a smooth and quick way to reach the specified target. Additionally, it illustrates that the nonlinear MPC method proposed is effective.

3) Generalization ability: The PSO-based SVM obtain good performance in all problems. This means that the optimization of the SVM parameters works well in different problems and the proposed method can utilized into other problems conveniently.

The experiment results verify the effectiveness of both PSO-based SVM prediction method and PSO-based rolling optimization method in the proposed model predictive control algorithm.

\section{Discussions and Conclusion}

In this paper a two-stage PSO-based nonlinear model pre- dictive control method is proposed to control the steel slab temperature in the reheating furnace. In this method PSO is firstly utilized to optimize the SVM parameters to improve the predictive model. Then, the PSO is utilized in rolling optimization section of the proposed nonlinear model predictive control method. The experiments based on real production data illustrate that these two optimization processes are effective to improve the control accuracy for different problems.

The optimization of the predictive model will take a long time, but this optimization process can be done off-line and the constructed model can be utilized on-line to control the reheating furnace as a real time control method. The proposed method provides a possible way to achieve the target of intelligent control in the reheating furnace temperature control problem. Besides, the proposed method can be utilized in other similar problem with the adjustment of the train data and the parameters of the proposed method.

In the future, we will focus on two directions: 1) the further improvement of prediction accuracy and control accuracy by utilizing other optimization methods; 2) the applications of the proposed method in other problems.

\section{Acknowledgement}

This research is partly supported by National Natural Science Foundation (Grant No. 61374207), National 863 High-Tech Research and Development Program of China (2013AA040704).

\section{REFERENCES}

1) Y. X. Liao, J. H. She and M. Wu: IEEE Trans. Ind. Electron., 59 (2009), No. 7, 2704.

2) L. X. Tang, G. S. Wang and Z. L. Chen: Oper. Res., 2014. (Doi: 10.1287/opre.2014.1278).

3) L. X. Tang, G. S. Wang, J. Y. Liu and J. Y. Liu: Naval Res. Logistics, 58 (2011), No. 4, 370.

4) L. X. Tang, J. Y. Liu, A. Y. Rong and Z. H. Yang: Eur. J. Oper. Res., 124 (2000), No. 2, 267.

5) Z. J. Wang, C. Shao and T. Y. Chai: Proc. IEEE Int. Conf. on Control Application, IEEE, Piscataway, NJ, (1999), 29.

6) Y. I. Kim, K. C. Moon, B. S. Kang, C. Han and K. S. Chang: Control Eng. Pract., 6 (1998), 1009.

7) H. S. Ko, J.-S. Kim, T.-W. Yoon, M. Lim, D. R. Yang and I. S. Jun: Proc. in American Control Conf., IEEE, Piscataway, NJ, (2000), 2725.

8) A. Steinboeck, D. Wild and A. Kugi: Control Eng. Pract., 21 (2013), 495.

9) H. Sibarani and Y. Samyudia: Comput-Aided Chem. Eng., 18 (2004), 811.

10) R. Rouhani and R. Mehra: Automatica, 18 (1982), No. 4, 401.

11) L. X. Tang and X. P. Wang: IEEE Trans. Evol. Comput., 17 (2013), No. 1, 20.

12) L. X. Tang, Y. Zhao and J. Y. Liu: IEEE Trans. Evol. Comput., 18 (2014), No. 2, 209

13) D. M. Prett, B. L. Remarker and C. R. Cutler: US4349869. A, (1979), 1.

14) D. W. Clarke, C. Mohtadi and P. S. Tuffs: Automatica, 23 (1987), No. 2, 137.

15) S. Mahmoodi, J. Poshtan, M. R. Jahed-Motlagh and A. Montazeri: Chem. Eng. J., 146 (2009), No.3, 328.

16) P. F. Tsai, J. Z. Chu, S. S. Jang and S. S. Shieh: J. Process Contr., 13 (2003), No. 5, 423.

17) V. Puig, G. Cembrano, J. Romera, J. Quevedo, B. Aznar, A. Ramon and J. Cabot: Water Sci. Technol., 60 (2009), No. 4, 869.

18) X. N. Zhang, D. M. Vilathgamuwa, K. J. Tseng, B. S. Bhangu and C. J. Gajanayake: IEEE Trans. Power Electron., 28 (2013), No. 12, 5804.

19) M. Jalili, S. Atashbari, S. Momenbellah and F. H. Roudsari: Int. J. Wavelets Multi., 5 (2007), No. 1, 69.

20) J. H. Jang, D. E. Lee, C. Kim and M. Y. Kim: ISIJ Int., 48 (2008), No. $10,1325$.

21) V. N. Vapnik: The nature of statistical learning theory, New York: Springer, (1995), 1.

22) J. Kennedy and R. C. Eberhart: IEEE Int. Conf. on Neural Networks, Vol. 4, IEEE, Piscataway, NJ, (1995), 1942. 